\title{
Emerging Insights and New Perspectives on the Nature of Hydrocephalus
}

\author{
Luke D. Tomycz ${ }^{a, b}$ Andrew T. Hale ${ }^{c}$ Timothy M. George ${ }^{a, b}$ \\ ${ }^{a}$ Division of Pediatric Neurosurgery, Dell Children's Medical Center, and ${ }^{b}$ Dell School of Medicine, University of \\ Texas at Austin, Austin, TX, and ' $M$ Medical Scientist Training Program, Vanderbilt University School of Medicine, \\ Nashville, TN, USA
}

\section{Introduction}

Like many disease conditions, our understanding of hydrocephalus has evolved over its long history. References to enlarged skulls can be found in ancient Egyptian texts dating back to 2,500 BC. Hippocrates would write about hydrocephalus in the 4th century BC and Galen would add to our understanding in the 2 nd century $\mathrm{AD}$ [1]. The first documented attempt to treat the condition would occur many centuries later in 1,000 AD by Abulcasis, an Arabian surgeon who described incising the scalp and draining fluid from within the head [2]. New innovations were slow to come however, and not until the Spitz-Holter shunt valve was developed in the mid-1950s was there any effective treatment for this hitherto relentless and fatal condition [3].

The classical dogma regarding the pathophysiology of hydrocephalus is well known by not only every practicing neurosurgeon, but by most students of medicine. Cerebrospinal fluid (CSF), created and secreted by active transport of the choroid plexus within the lateral, third, and fourth ventricles, travels by bulk flow toward the arachnoid granulations where passive reabsorption occurs. Hydrocephalus is overwhelmingly a problem of CSF malabsorption (i.e., "communicating hydrocephalus") caused by any of a variety of initial insults (e.g., infection, hemorrhage) that somehow impair the function of the arachnoid granulations to properly reabsorb fluid. Alternatively, an anatomic blockage of a portion of the ventricular pathway

\section{KARGER}

(C) 2017 S. Karger AG, Basel

E-Mail karger@karger.com

www.karger.com/pne (e.g., cyst, tumor) can cause an "obstructive hydrocephalus" in which the reabsorption mechanisms are presumably intact, but bulk flow is blocked and the ventricles dilate proximal to the obstruction. In both variants, ventricular dilation is thought to be the end result of hydrostatic pressure build-up within the ventricles.

Though perhaps intuitive, the preceding narrative of CSF physiology and pathophysiology is surprisingly thinly supported by empirical evidence. Furthermore, recent investigations, both in the realm of basic science using animal models and radiological sciences using advanced imaging techniques (e.g., cine phase-contrast MRI), have directly challenged many of the underlying assumptions of the classical dogma. Belgian scientist Georges LeMaitre, the physicist who first proposed the Big Bang theory, recognized that even a single experiment that yielded observations inconsistent with the fundamental claims of the theory risked invalidating the entire hypothesis [4]. At the very least, such an experiment would mandate a modification of the theory to account for the new and discordant observations. Only recently has the neurosurgical community begun to apply the same level of scientific rigor to the study of hydrocephalus.

In a 2006 report by Bergsneider et al. [5], the provocative and important question was posed: "... is there more to hydrocephalus than our long-held and oversimplified concept of it as a plumbing problem of mismatched CSF production and resorption...?" Asking questions and challenging old beliefs is only the first step. But it is the

Luke Tomycz, MD

1301 Barbara Jordan Boulevard 307

Austin, TX 78723 (USA)

E-Mail tomyczluke@gmail.com 
critical first step. New investigations (and answers) will follow only once the bold admission is made that the old theories can no longer be maintained under more intensive scrutiny. In that same spirit, we critically analyze the scientific evidence that supports various claims, and recent observations that seem to run contrary to prevailing notions. New ideas in science are not typically the result of an old idea being gradually and incrementally modified. Instead, the history of scientific discovery is a history of paradigm shifts that dramatically usher in novel perspectives, suggesting new directions for exploration and promising new horizons.

\section{A Brief History of Hydrocephalus}

\section{Walter Dandy's Hydrocephalus}

Walter Dandy developed experimental models of hydrocephalus in the early part of the 20th century. In a single experiment on a dog (which was later attempted by several investigators but never reproduced), the foramen of Monro was occluded and unilateral choroid plexectomy was performed. This led to dilation of the contralateral lateral ventricle and collapse of the plexectomized ventricle. Based on this, Dandy concluded with "absolute proof" that the choroid plexus is the primary site of CSF production [6]. This led to several attempted treatments of hydrocephalus that involved surgical choroid plexectomy, but the results were overwhelmingly disappointing [7, 8]. Dandy's experiments further seemed to indicate that CSF was not absorbed primarily within the ventricles, implicating an extraventricular site for CSF resorption. Dandy was also among the first to classify hydrocephalus as either "communicating" or "noncommunicating" (i.e., "obstructive") based on one's ability to recover a supravital dye injected in the ventricles from the lumbar subarachnoid space [9]. Unlike communicating hydrocephalus, which Dandy felt he could not adequately explain, obstructive hydrocephalus provided an opportunity for treatment via third ventriculostomy that at the time was viewed as a sort of internal shunt bypassing the site of intraventricular obstruction.

\section{The Contributions of Harvey Cushing}

The bulk flow model of CSF - the idea that CSF flowed through the neuraxis from the site of creation within the ventricles, through the lumbar cisterns, and to a point of resorption in the surrounding cisterns - was actually posited by Harvey Cushing as early as 1925 in his work "The
Third Circulation" [10]. At the time, it was a radical departure from the "ebb and flow" model that was dominant among contemporaries. Harvey Cushing, working with Weed, can also be credited for popularizing the idea of the arachnoid granulations as the site of CSF resorption. Weed reproduced and modified earlier experiments of Key and Retzius in which a gelatin solution colored with Berlin blue dye was injected into the lumbar subarachnoid space in human cadavers and observed to pass through the arachnoid granulations into the venous sinuses as well as through lymphatic vessels into the frontal sinus and nasal membranes [6]. Interestingly, Dandy was skeptical and challenged the concept of specialized structures being invoked to explain fluid resorption in the brain that seemed to occur in a similar fashion in other body cavities (e.g., pleura, peritoneum). As early as 1914, he conducted experiments in which a colored tracer was injected intrathecally and recovered in the urine, supporting the claim that CSF is reabsorbed via microvessels throughout the subarachnoid space [11]. Ultimately, however, the narrative advanced by Cushing found its way into the mainstream, and subsequently into the neurosurgical textbooks. Additional contributions were later recognized both in CSF production (i.e., from the ependymal surface cells) and in CSF reabsorption (i.e., via perineural lymphatic routes), but these pathways were deemed to be minor under normal, physiologic conditions.

\section{Defining and Classifying Hydrocephalus}

Hydrocephalus, as we currently use the term, can be applied to a vast array of pathologies. It can occur congenitally without a recognizable antecedent or in the premature infant following neonatal meningitis or germinal matrix hemorrhage. It can occur at any age. It can occur in conjunction with ventriculomegaly, but also in the context of normal-sized or even slit ventricles. It is found typically in the setting of elevated intracranial pressure (ICP), but can also be seen in patients with relatively normal pressures and, rarely, even low pressures. Hydrocephalus can last for days or plague an individual throughout his or her entire life.

In the 1960s Ransohoff et al. [12] declared that all hydrocephalus is obstructive. Communicating hydrocephalus merely represented an obstruction at the site of the arachnoid granulations of the venous sinuses. Rekate [13] rephrased this basic idea in 2008, defining hydrocephalus as "an active distention of the ventricular system of the
362

Pediatr Neurosurg 2017;52:361-368 DOI: $10.1159 / 000484173$
Tomycz/Hale/George 
brain resulting from inadequate passage of CSF from its point of production within the ventricles to its point of resorption into systemic circulation." Intrinsic to these classifications was the assumption that bulk flow of CSF was somehow disrupted in hydrocephalus, again either from a mass obstructing the ventricles or from impaired resorption; brain pulsations were not considered. Heeding the intracranial pulsatility experiments of Bering [14, 15] and Di Rocco et al. [16], and making observations about CSF flow from cine phase-contrast MRI, a radiologist named Greitz [17] introduced a hydrodynamic theory dividing hydrocephalus into acute (i.e., obstructive) and chronic hydrocephalus. Chronic hydrocephalus, rather than being related to CSF malabsorption, was felt to be associated with: (1) decreased intracranial compliance, and (2) increased pulse pressure within brain capillaries, leading to ventricular enlargement. Madsen et al. [18] proposed a "fourth circulation" of intracranial pulsatility, coursing with each heartbeat from the arterial to venous sides of the brain.

Increasingly, experts are recognizing the heterogeneity inherent in hydrocephalus, and conducting trials that focus on subtypes of hydrocephalus (e.g., outcome of endoscopic third ventriculostomy [ETV] in infants with myelomeningocele) rather than lumping together every patient with symptomatic intracranial fluid accumulation [19]. The etiology of hydrocephalus resulting from intracranial infection or trauma might differ significantly from the hydrocephalus in a child with a fourth ventricular tumor, and the optimal treatment approaches may vary accordingly. Indeed, some of the difficulty in defining hydrocephalus may be the result of this haphazard clustering of numerous, disparate entities (e.g., normal pressure hydrocephalus [NPH], benign hydrocephalus of infancy, posthemorrhagic hydrocephalus, idiopathic hydrocephalus, pseudotumor cerebri) into the same category based on some superficial similarities in presentation, imaging findings, and treatment. Without detailing an exhaustive review of all the controversies surrounding the contemporary understanding of CSF physiology and hydrocephalus, the following sections should compel the reader to question old assumptions and entertain alternative models.

\section{The Site and Rate of CSF Production}

The glandular appearance of the choroid plexus in the ventricles made it natural to consider this as the site of fluid production within the ventricles. The failure of bi-

Emerging Insights and New Perspectives

on the Nature of Hydrocephalus lateral extirpation of the choroid plexus to benefit patients with hydrocephalus, however, suggested the possibility that extrachoroidal CSF production played a significant role [20]. Experiments by Milhorat [21] in 1969 demonstrated progressive dilation of isolated, plexectomized ventricles clearly depicting the nonessential nature of the choroid plexus in CSF production. The chemical composition of CSF was also studied in plexectomized patients and found to be unchanged [22]. While classic dogma holds that CSF formation is an active process, independent of intracranial pressure, multiple experiments show that CSF formation actually declines with increased pressure, and that CSF formation may in fact be a passive process [23]. CSF can be observed in the neural tubes of fetal pigs and humans before the choroid plexus anlage appears, and CSF has been appreciated in the ventricular cavities of some lower vertebrates that altogether lack a choroid plexus [24]. Whatever the role of the choroid plexus, it can be stated unequivocally that it is not essential for life, and if removed, the individual will typically continue to survive with more or less the same amount and composition of CSF.

The rate of CSF formation in adult humans is generally quoted to be $0.3-0.4 \mathrm{~mL} / \mathrm{min}$. It is instructive to review the experiments that yielded this value, many of which were conducted nearly 100 years ago, and have since been criticized for improper assumptions and poor methodology. The most important and frequently referenced experiment to arrive at a CSF formation rate was the ventriculocisternal perfusion experiment or so-called Pappenheimer technique. Oreskovic et al. [25] offer a detailed description of the methodology employed as well as a sobering critique of the conclusions. Essentially, by casting doubt on the numerous assumptions of the ventriculocisternal perfusion experiment - (1) CSF is created by the choroid plexus only, not everywhere throughout the neuroaxis, (2) CSF and perfusate is absorbed only by arachnoid granulations within the cisterns, and nowhere within the ventricular system, and (3) CSF flows in a linear, bulk fashion rather than being agitated and mixed by pulsations - the calculations based on these assumptions become increasingly difficult to accept.

An elegant experiment conceived by neurosurgeons in Zagreb involved direct surgical cannulation of the aqueduct of Silvius. Intended to capture the net production of the lateral ventricles and third ventricle, this experiment yielded a surprising result: after several hours of observation, CSF pulsated near the tip of the cannula, but no fluid was collected [24]. These findings suggested that the net CSF formation within an isolated ventricle, under

Pediatr Neurosurg 2017;52:361-368 363 
normal physiologic conditions, is unmeasurably small. In other words, CSF created within the ventricle was essentially equivalent to CSF resorbed within the same ventricle. No net fluid was collected after cannulation of the aqueduct for several hours. This result was corroborated by at least 2 separate cine phase-contrast MRI studies, which demonstrated an aqueduct stroke volume (ASV) of essentially zero $[26,27]$. The invasive nature of aqueductal cannulation and the lack of reproducibility inherent in flow-sensitive MR techniques continues to stoke controversy as to the degree of confidence in these results.

\section{CSF Resorption and Lymphatic Clearance Pathways}

A significant problem for the proposal that arachnoid granulations are the primary site of CSF absorption is the fact that human arachnoid villi are not present at birth (or at any time in utero) $[28,29]$. Most of the studies that focus on the role of the human arachnoid granulations center on electron microscopic studies that describe "tubular structures with dual openings" and go on to loosely postulate how these structures may be involved in CSF transport. A great deal of contemporary research focuses on arachnoid cap cells, a layer of cells at the apical portion of the granulation unique to humans which is in contact with both CSF and venous blood [30]. A thorough and relatively recent review of CSF outflow mechanisms admits that since much of the existing literature focuses on animal models and emphasizes "anatomic and morphologic characteristics (of arachnoid granulations)," current understanding of the "functional attributes" of CSF outflow physiology in humans is "incomplete and poorly understood" [31]. In terms of conclusive scientific evidence (either in animals or in humans) that can clearly elucidate a mechanism for this proposed fluid transfer from the CSF cisterns into the venous system via arachnoid granulations - at this point, it simply does not exist.

The discovery that astrocytes express aquaporins (i.e., transmembrane water channels) has led to the concept that water transport might occur continuously between cerebral arteries, CSF spaces, and interstitial fluid (ISF) compartments of the brain [30]. As early as 1952, instillation of radiolabeled water (alternatively referred to as "heavy water" or deuterium oxide) into the brain led to a rapid distribution throughout all brain compartments. At least 6 different aquaporin channels have been described in human brain tissue, and there is evidence that the permeability and localization of these channels can undergo modifications in disease states (e.g., brain tumors, injured brain) on the order of minutes and even seconds [32]. Recent findings have compelled "... a growing number of researchers to reach the consensus that ISF and CSF are mainly formed and reabsorbed across the walls of CNS blood capillaries, which implies there is no need for a directed CSF circulation from the choroid plexus to the arachnoid villi" [30]. The radiologic finding of "transependymal" flow could potentially be better explained by a model in which CSF continuously shifts between ventricles, subarachnoid spaces, and the brain interstitium.

In order to characterize CSF resorption and understand CSF flow patterns, numerous studies have utilized a variety of large molecules as CSF substrates. However, since CSF is $99 \%$ composed of water, and aquaporins have been shown to play a prominent role in intracranial water transfer, it has been questioned whether these large molecules are appropriate markers for CSF turnover. Bulat et al. [33] compared the pathway of radiolabeled water to that of the large nonabsorbable polysaccharide, inulin, after injection into the lateral ventricles in cats. Not surprisingly, divergent pathways clear these substances after injection into a brain ventricle at normal pressures. In this experiment, the concentration of radiolabeled water, measured hours after intraventricular instillation, increased in the venous system and remained undetectable within the cisternal CSF, suggesting an intraventricular site for CSF resorption under normal conditions. The cisternal concentration of inulin, on the other hand, increased sharply after $3 \mathrm{~h}$ due either to bulk flow or diffusion aided by pulsatile agitation. Based on these findings, the authors suggested a new hypothesis of CSF physiology: "... [CSF] appears and disappears throughout the entire CSF system depending on hydrostatic and osmotic forces between the CSF, ISF and blood capillaries. In terms of the capacity of fluid exchange, the cerebral capillaries are the dominant location ... [33, 34]." Despite criticism that this model oversimplifies the complex regulation of CSF turnover at the blood-brain barrier, these findings at least mandate a reconsideration of the established dogma.

In terms of the lymphatic component of CSF outflow, numerous animal studies have detailed the injection of a variety of compounds into the CSF system (e.g., Berlin blue dye, carbon particles, gold particles, India ink and other inks, silicon particles) and documented their excretion via lymphatic pathways. While "open-cuffed" and "closed-cuffed" models offer some insight into how CSF itself might be drained by lymphatic capillaries, the actual mechanism remains poorly understood [30]. Jessen et al. [35] stimulated a renewed interest in the Virchow- 
Robin spaces (VRS) of the brain. Instillation of fluorescent tracers of varying molecular weight into the cisterna magna led to a rapid concentration of fluorescence within the VRS, from where the smaller tracers passed into the interstitium of the brain. These findings led to the concept of a previously unknown system for the clearance of interstitial protein waste in the brain, and the authors called it the "glymphatic system." The sluggishness of flow within the VRS combined with the technical difficulty of these experiments makes it challenging to replicate results and continues to foster fierce debate about the importance of this new pathway and the directionality of flow.

\section{The Third Circulation}

Dandy's 100-year-old experiment implicated the choroid plexus as the primary site of CSF formation and suggested an extraventricular site of CSF reabsorption [11]. By necessity, like a slow river, CSF would have to flow from the ventricles through the various foramen and aqueducts into the surrounding subarachnoid cisterns where it would be reabsorbed. How it flowed into the lumbar cistern, and then circulated back up into the head against gravity, always prompted controversy, consternation, and some amount of befuddlement. Particle tracer and dye studies seemed to bolster the slow-river, bulk flow model as various perfusates infused into the ventricles could later be recovered in the lumbar cisterns and vice versa [34]. Of course, without any element of bulk flow, a similar redistribution of iodinated contrast, for example, could be expected based on the simple principles of diffusion aided by pulsatile agitation and mixing.

The direct aqueductal cannulation experiment mentioned previously not only yielded insights about the nature of CSF formation and resorption, but also offered a new perspective on CSF bulk flow. After watching fluid bobbing within the cannula for multiple hours, the authors suggested that bulk flow of CSF throughout the ventricular system is perhaps a marginal component of total CSF motion [19]. Cine phase-contrast MRI studies have demonstrated that the kinetic energy of CSF circulation is overwhelmingly from pulsatile motion, rather than bulk flow $[36,37]$. Bulk flow and diffusional mixing may be more important for the transfer of large molecules in the CSF (e.g., immunoglobulins, hormones, cells, etc.), whereas the turnover of CSF is a more local phenomenon.

Emerging Insights and New Perspectives on the Nature of Hydrocephalus

\section{What Is the Mechanism for Ventriculomegaly?}

The classical theory of hydrocephalus holds that the ventricles enlarge because of either an intraventricular blockage or impaired fluid resorption resulting in CSF accumulation and elevated hydrostatic pressure within the ventricle. However, when the ventricular space is in communication with the subarachnoid space, the development of such a "transmantle pressure gradient" runs contrary to Laplace's law. Indeed, numerous experimental studies fail to demonstrate a difference in pressure - either in normal subjects or in animal models with hydrocephalus - between the ventricles and the subarachnoid space [18]. While some posit that the measuring devices may lack the sophistication to appreciate the small and transient, transmantle pressure gradient that precedes the development of ventriculomegaly, the more plausible argument is that no pressure gradient exists and rather something other than a hydrostatic pressure differential needs to be invoked to describe the phenomenon of ventricular enlargement. Indeed, many examples exist clinically and anecdotally during which ventricular expansion occurs without particularly high pressures (e.g., some shunt malfunctions, NPH, low-pressure hydrocephalus). Other detractors from the classical descriptions of ventriculomegaly point out that an obstruction to CSF resorption at the arachnoid granulations within the cisterns should lead to cisternal subarachnoid fluid accumulation rather than the observed ventricular enlargement and subarachnoid space crowding.

As early as 1943, O'Connell postulated that increased CSF pulse pressure could be the cause of communicating hydrocephalus [38]. The experiments of Bering and Di Rocco in the 1960s and 1970s offered experimental evidence supporting this alternative explanation for ventriculomegaly: aberrant distribution of intracranial pulsatility. Bering [15] revealed that unilateral choroid plexectomy and/or unilateral anterior choroidal artery embolization could lead to an ipsilateral decrease in lateral ventricular size in animal models of communicating hydrocephalus; pressure monitors in both ventricles showed a decrease in the pulse pressure (peak to trough differential) on the side of the embolization despite the mean hydrostatic pressure being equal between the ventricles. Later, Di Rocco et al. [16] took normal animals and experimentally induced an isolated ventriculomegaly by implanting small balloons that inflated and deflated with each heartbeat, changing the pulsatile dynamics within the implanted ventricle. Taken together, the implications of these experiments are astounding. While these

Pediatr Neurosurg 2017;52:361-368 DOI: $10.1159 / 000484173$ 
experiments should have raised significant questions within the neurosurgical community about the widely accepted, classical theories of hydrocephalus and ventriculomegaly, they instead went largely unnoticed. Only years later, when ventriculocisternostomy (i.e., ETV) was being used successfully in patients with so-called communicating hydrocephalus, did neurosurgeons again begin to turn their attention to the role of intracranial pulsations in CSF hydrodynamics.

\section{Intracranial Pulsations}

The brain is a pulsatile organ. Pulsatility is a feature of intracranial pressure, which although often relayed with a single numeric value, is actually a complex waveform, as demonstrated by ICP monitoring. Pulsatility is also a feature of CSF flow, as cine phase-contrast MRI has demonstrated both in the cranial/caudal pulsation of flow within the aqueduct of Silvius, as well as in subarachnoid/ cisternal compartments [18]. Pressure pulsatility and flow pulsatility are related in a complex fashion that is further a function of time, precise location, and brain compliance. While respiratory- and vasomotor-induced oscillations contribute to brain pulsatility, the main component is cardiovascular-induced pulsatility [17].

Several preclinical and clinical studies employing various measurement modalities (i.e., ICP monitoring, TCD, and cine phase-contrast MRI) have documented alterations in brain pulsatility in hydrocephalic states, as well as changes in the timing of propagation and morphology of the pulse waveform [39]. Eide and Sorteberg [40] demonstrated that CSF shunting modifies not only mean pressure but also pulse pressure, and that in NPH, presurgical pulse pressure may offer insight into the likelihood of responsiveness to shunting. While ventriculomegaly and resulting compression of cisternal and convexity subarachnoid spaces increases impedance to pulsatility in the subarachnoid space and only worsens the pulsatility maldistribution, shunt implantation decreases ventricular size and opens up the compressed subarachnoid space, perhaps increasing conductance of pulsatility through this area. Animal models of hydrocephalus typically exhibit hyperdynamic flow within the ventricular system, manifest with increased ASV. However, a weak correlation between ventricular volume and ASV has led others to investigate whether the ratio of ASV to cervical subarachnoid stroke volume might not be a better indicator of developing hydrocephalus [41]. In other words, rather than a static transmantle pressure gradient (which has never been demonstrated) causing ventriculomegaly, perhaps it is a pulsatile pressure gradient (e.g., increased pulsatility within the ventricles relative to the subarachnoid space), which correlates with increased ventricular size [16]. Using cine phase-contrast MRI, Greitz [17] demonstrated an increased ASV and decreased pulsatile flow at $\mathrm{C} 2$ in patients with $\mathrm{NPH}$, although subsequent studies found no such gradient [42].

ETV began to attract renewed attention when it was being used effectively in patients with $\mathrm{NPH}$, myelomeningocele, and other forms of so-called communicating hydrocephalus [19]. While ETV as a bypass for aqueductal stenosis or a fourth ventricular outlet obstruction seemed intuitive, the classical theory of hydrocephalus offered no explanation as to why ETV seemed to resolve symptoms in countless patients with nonobstructive hydrocephalus. Invoking aberrant pulsatility as a mechanism for ventriculomegaly, however, led to a new set of speculations. Perhaps by reconnecting the ventricular space with the subarachnoid cisterns, ETV acts as a pulsation absorber and effects a redistribution of pulsatile energy from the ventricular space to the subarachnoid space. Warf [43] hypothesized that choroid plexus cauterization potentiates this effect by eliminating the choroid plexus, thereby neutralizing the source of ventricular pulsatility. Many are shifting their focus from the effects of shunts on bulk flow and the potential role of ETV as an internal bypass, and instead trying to understand the changes caused by these treatments on intracranial pulsatility and brain compliance. Certainly, more experiments are needed as well as an ability to correlate noninvasive measurements of flow pulsatility (e.g., flow-sensitive MRI, TCD) with invasive pressure monitoring.

\section{The Way Forward}

The model of hydrocephalus that is beginning to take form in light of recent experimental revelations is a dramatic departure from the classical theory of hydrocephalus. Numerous observations from animal experiments, clinical series, and advanced imaging techniques challenge some of the most basic assumptions we have about CSF physiology: the site and rate and mechanism of CSF formation and resorption, the etiology of ventricular dilation, the nature of CSF flow, the pathologic underpinnings of hydrocephalus, the mechanism and action of CSF shunting and third ventriculostomy. Even the longheld classification of "communicating" and "obstructive" hydrocephalus seems insufficiently simplified, based on 
inaccurate assumptions, and no longer particularly instructive in terms of guiding treatment. It is frankly becoming untenable to continue to support the classic description of hydrocephalus as "a plumbing problem of mismatched CSF production and resorption.”

As pediatric neurosurgeons become increasingly aggressive in trying to achieve shunt independence for patients, we continue to learn more about the pathophysiology of this disease and how our treatments might be having an effect. Notwithstanding the countless lives saved by shunts, we must view them honestly for what they are: devices that do not cure, but rather allow patients to live with hydrocephalus, albeit under the perpetual sentence of a shunt, all too prone to obstruction, infection, occlusion, disconnection, and other forms of malfunction. But as we become more critical of classic experiments of CSF physiology, we must reserve a similar skepticism for new research until methodological rigor and reproducibility establish findings with a high level of confidence. We look with optimism toward a future where a clearer understanding of why hydrocephalus occurs will yield more definitive treatments and a better quality of life for millions of affected patients.

\section{Preview of the Special Edition on Hydrocephalus}

The Evolution of Cerebrospinal Fluid Shunts: Advances in Technology and Technique

Tomei provides a highly practical and comprehensive review on different types of CSF shunts, catheters, and valves, as well as surgical techniques to avoid shunt failure.
Cerebrospinal Fluid Shunting Complications in Children

Hanak et al. discuss their research into precisely what causes the obstruction of catheters implanted in the brain, and the implications for how shunts might be better designed.

The Global Rise of Endoscopic Third Ventriculostomy with Choroid Plexus Coagulation for Pediatric Hydrocephalus

Dewan et al. consider the reemergence of endoscopic third ventriculostomy with choroid plexus coagulation as an innovation born of necessity in Uganda.

Aquaporin Water Channels and Hydrocephalus

Verkman et al. share new perspectives on aquaporins in the brain and the role they play in water transfer, specifically focusing on the development of hydrocephalus.

New Concepts of Cerebrospinal Fluid Physiology and

Development of Hydrocephalus

Oreskovic et al. report on the extensive animal research they have done on CSF turnover and bulk flow, and propose new models for CSF physiology and intracranial hydrodynamics.

Cerebrospinal Fluid Biomarkers of Pediatric Hydrocephalus

Limbrick et al. offer insight into the use of biomarkers to classify, detect, and possibly treat patients with hydrocephalus and shunt malfunction.

\section{Advanced Neuroimaging Techniques in Pediatric}

Hydrocephalus

Patel et al. use advanced imaging techniques to describe functional and ultrastructural changes observed in the hydrocephalic brain.

Neural Stem Cells and Fetal-Onset Hydrocephalus

Rodriguez and Guerra discuss the role of aberrant neurodevelopment in fetal and congenital hydrocephalus, and suggest the possibility of using stem cells as treatment.

\section{References}

1 Dimopoulos VG, Robinson JS, Fountas KN: The pearls and pitfalls of skull trephination as described in the hippocratic treatise "On Head Wounds.” J Hist Neurosci 2008; 17:131140.

2 Abu al-Qasim Khalaf ibn 'Abbas al-Zahrawi: Albucasis: On Surgery and Instruments: A Definitive Edition of the Arabic Text with English Translation and Commentary by MS Spink and GL Lewis. London, Wellcome Institute of the History of Medicine, 1973.

3 Boockvar JA, Loudon W, Sutton LN: Development of the Spitz-Holter valve in Philadelphia. J Neurosurg 2001;95:145-147.

4 Lemaitre G: Republication of: a homogeneous universe of constant mass and increasing radius accounting for the radial velocity of extra-galactic nebulae. Gen Relat Gravit 2013; 45:1635-1646.

Emerging Insights and New Perspectives on the Nature of Hydrocephalus
5 Bergsneider M, Egnor MR, Johnston M, Kranz D, Madsen JR, McAllister JP, Stewart C, Walker ML, Williams MA: What we don't (but should) know about hydrocephalus. J Neurosurg 2006;104:157-159.

6 Canale DJ, Longo LD: Harvey Cushing and pediatric neurosurgery. Neurosurgery 1990; 27:602-611.

7 Milhorat TH: Failure of choroid plexectomy as treatment for hydrocephalus. Surg Gynecol Obstet 1974;139:505-508.

8 Van den Bergh R, Beuls E: Plexectomy in the management of hydrocephalus. Eur Neurol 1972;8:286-295.

9 Milhorat TH: The third circulation revisited. J Neurosurg 1975;42:628-645.

10 Cushing H: Studies in intracranial physiology and surgery; the third circulation, the hypophysics, the gliomas. London, Milford/Oxford University Press, 1926.
11 Dandy W, Blackfan KD: Internal hydrocephalus: an experimental clinical and pathological study. Am J Dis Child 1914;8:406-482.

12 Ransohoff J, Shulman K, Fishman RA: Hydrocephalus: a review of etiology and treatment. J Pediatr 1960;56:399-411.

13 Rekate HL: The definition and classification of hydrocephalus: a personal recommendation to stimulate debate. Cerebrospinal Fluid Res 2008;5:2.

14 Bering EA Jr: Choroid plexus and arterial pulsation of cerebrospinal fluid: demonstration of the choroid plexuses as a cerebrospinal fluid pump. AMA Arch Neurol Psychiatry 1955; 73:165-172.

15 Bering EA Jr: Circulation of the cerebrospinal fluid: demonstration of the choroid plexuses as the generator of the force for flow of fluid and ventricular enlargement. J Neurosurg 1962;19:405-413. 
16 Di Rocco C, Pettorossi VE, Caldarelli M, Mancinelli R, Velardi F: Communicating hydrocephalus induced by mechanically increased amplitude of intra-ventricular cerebrospinal-fluid pressure: experimental studies. Exp Neurol 1978;59:40-52.

17 Greitz D: Radiological assessment of hydrocephalus: new theories and implications for therapy. Neurosurg Rev 2004;27:145-167.

18 Madsen JR, Egnor M, Zou R: Cerebrospinal fluid pulsatility and hydrocephalus: the fourth circulation. Clin Neurosurg 2006;53:48-52.

19 Teo C, Jones R: Management of hydrocephalus by endoscopic third ventriculostomy in patients with myelomeningocele. Pediatr Neurosurg 1996;25:57-63.

20 Lorenzo AV, Page LK, Watters GV: Relationship between cerebrospinal fluid formation, absorption and pressure in human hydrocephalus. Brain 1970;93:679-692.

21 Milhorat TH: Experimental hydrocephalus. 1. A technique for producing obstructive hydrocephalus in monkey. J Neurosurg 1970;32: 385-389.

22 Go KG, Koster-Otte L, Pratt JJ: Brain sodium uptake after choroid plexectomy. Brain Res 1979; 170:325-331.

23 Lanman RC, Schanker LS: Transport of choline out of the cranial cerebrospinal fluid spaces of the rabbit. J Pharmacol Exp Ther 1980;215:563-568.

24 Oreskovic D, Klarica M: The formation of cerebrospinal fluid: nearly a hundred years of interpretations and misinterpretations. Brain Res Rev 2010;64:241-262.

25 Oreskovic D, Klarica M, Vukic M, Marakovic $\mathrm{J}$ : Evaluation of ventriculo-cisternal perfusion model as a method to study cerebrospinal fluid formation. Croat Med J 2003;44:161-164.

26 Battal B, Kocaoglu M, Bulakbasi N, Husmen G, Tuba Sanal H, Tayfun C: Cerebrospinal fluid flow imaging by using phase-contrast MR technique. Br J Radiol 2011;84:758-765.
27 Scollato A, Gallina P, Gautam B, Pellicano G, Cavallini C, Tenenbaum R, Di Lorenzo N: Changes in aqueductal CSF stroke volume in shunted patients with idiopathic normalpressure hydrocephalus. Am J Neuroradiol 2009;30:1580-1586.

28 Kuruvilla LC: Benign enlargement of subarachnoid spaces in infancy. J Pediatr Neurosci 2014;9:129-131.

29 Nickel RE, Gallenstein JS: Developmental prognosis for infants with benign enlargement of the subarachnoid spaces. Dev Med Child Neurol 1987;29:181-186.

30 Brinker T, Stopa E, Morrison J, Klinge P: A new look at cerebrospinal fluid circulation. Fluids Barriers CNS 2014;11:10.

31 Kapoor KG, Katz SE, Grzybowski DM, Lubow M: Cerebrospinal fluid outflow: an evolving perspective. Brain Res Bull 2008;77:327-334.

32 Filippidis AS, Kalani MY, Rekate HL: Hydrocephalus and aquaporins: lessons learned from the bench. Childs Nerv Syst 2011;27:2733.

33 Bulat M, Lupret V, Oreskovic D, Klarica M: Transventricular and transpial absorption of cerebrospinal fluid into cerebral microvessels. Coll Antropol 2008;32:43-50.

34 Ozisik P, Roth J, Beni-Adani L, Constantini S: Continuous spinal drain following endoscopic third ventriculostomy: a proposal to change the definition of failure. Childs Nerv Syst 2011;27:1973-1978.

35 Jessen NA, Munk AS, Lundgaard I, Nedergaard M: The glymphatic system: a beginner's guide. Neurochem Res 2015;40:2583-2599.

36 Katayama S, Asari S, Ohmoto T: Quantitative measurement of normal and hydrocephalic cerebrospinal fluid flow using phase contrast cine MR imaging. Acta Med Okayama 1993; 47:157-168.

37 Quencer RM, Post MJ, Hinks RS: Cine MR in the evaluation of normal and abnormal CSF flow: intracranial and intraspinal studies. Neuroradiology 1990;32:371-391.
38 O'Connell JEA: The vascular factor in intracranial pressure and the maintenance of the cerebrospinal fluid circulation 1. Brain 1943; 66:204-228

39 Wagshul ME, Eide PK, Madsen JR: The pulsating brain: a review of experimental and clinical studies of intracranial pulsatility. Fluids Barriers CNS 2011;8:5.

40 Eide PK, Sorteberg W: Changes in intracranial pulse pressure amplitudes after shunt implantation and adjustment of shunt valve opening pressure in normal pressure hydrocephalus. Acta Neurochir 2008;150:11411147.

41 Scollato A, Tenenbaum R, Bahl G, Celerini M, Salani B, Di Lorenzo N: Changes in aqueductal CSF stroke volume and progression of symptoms in patients with unshunted idiopathic normal pressure hydrocephalus. AJNR Am J Neuroradiol 2008;29:192-197.

42 Baledent O, Gondry-Jouet C, Meyer ME, De Marco G, Le Gars D, Henry-Feugeas MC, IdyPeretti I: Relationship between cerebrospinal fluid and blood dynamics in healthy volunteers and patients with communicating hydrocephalus. Invest Radiol 2004;39:45-55.

43 Warf BC: Congenital idiopathic hydrocephalus of infancy: the results of treatment by endoscopic third ventriculostomy with or without choroid plexus cauterization and suggestions for how it works. Childs Nerv Syst 2013; 29:935-940.

44 Shapiro K, Marmarou A, Shulman K: Characterization of clinical CSF dynamics and neural axis compliance using the pressure-volume index. I. The normal pressure-volume index. Ann Neurol 1980;7:508-514.

45 Iliff JJ, Wang M, Zeppenfeld DM, Venkataraman A, Plog BA, Liao Y, Deane R, Nedergaard $\mathrm{M}$ : Cerebral arterial pulsation drives paravascular CSF-interstitial fluid exchange in the murine brain. J Neurosci 2013;33:1819018199. 